\title{
Impact of Combination Medical Therapy on Mortality in Vascular Surgery Patients
}

\author{
Thomas W. Barrett, MD, MCR ${ }^{1,2}$ \\ Kathleen Newton, $\mathrm{BS}^{3}$ \\ Caroline Koudelka, $\mathrm{MPH}^{4}$ \\ Motomi Mori, $\mathrm{PhD}^{3,4}$ \\ LeAnn Radcliffe, вs $^{5}$
}

\author{
${ }^{1}$ Section of General Medicine, Portland Veterans Affairs (VA) Medical Center, Portland, Oregon. \\ ${ }^{2}$ Division of Hospital Medicine, Oregon Health and Science University, Portland, Oregon. \\ ${ }^{3}$ Division of Biostatistics, Department of Public Health and Preventive Medicine, Oregon Health and Science \\ University, Portland, Oregon. \\ ${ }^{4}$ Center for Biostatistics Computing and Informatics in Biology and Medicine, Oregon Health and Science \\ University, Portland, Oregon. \\ ${ }^{5}$ Portland VA Research Foundation, Portland VA Medical Center, Portland, Oregon.
}

T.W.B. was supported, in part, by the Office of Research and Development, U.S. Department of Veterans Affairs, and by the Oregon Health and Science University Medical Research Foundation. Funds supported the data extraction and statistical analysis. The funding source had no role in the design, conduct, and analysis of the study, or in the decision to submit the manuscript for publication. T.W.B had full access to the data. Biostatistics support was provided in part by the Oregon Clinical and Translational Research Institute (NIH/NCRR UL1 RR024140).

This study was presented at the 3rd Annual Perioperative Medicine Summit, Cleveland Clinic, Cleveland, OH, September 11, 2007.

Disclosure: No conflicts of interest were reported.

BACKGROUND: Th use of beta-blockers or statins has been associated with decreased mortality after noncardiac surgery. There are no prior perioperative studies of concurrent use of other cardioprotective drugs.

OBJECTIVE: To ascertain whether combinations of aspirin, beta-blockers, statins, and/or angiotensin-converting enzyme (ACE) inhibitors were associated with decreased mortality 6 months after vascular surgery.

PATIENTS AND DESIGN: We performed a retrospective cohort study on the 3020 patients who underwent vascular surgery between January 1998 and March 2005 at 5 regional Veterans Affairs (VA) medical centers. The Cochran-Mantel-Haenszel test was used to assess associations with 6-month all-cause mortality for the combination drug exposures compared to no exposure while adjusting for propensity score.

RESULTS: Exposure to all 4 of the study drugs compared to none had a propensity-adjusted relative risk (aRR) of 0.52 (95\% confidence interval $[\mathrm{CI}], 0.26-1.01 ; P=0.052$ ), number needed to treat (NNT) 19; 3 drugs vs. none, aRR 0.60 (95\% CI, 0.38-0.95; $P=0.030$ ), NNT 38; 2 drugs vs. none, aRR 0.68 (95\% CI, 0.46-0.99; $P=0.043$ ), NNT 170; and 1 drug vs. none, aRR 0.88 (95\% CI, 0.63-1.22; $P=0.445$ ). ACE inhibitor exposure was common in all combinations.

CONCLUSIONS: Combination use of 2 to 3 study drugs, some of which included ACE inhibitors, was associated with decreased mortality after vascular surgery. Combination use of all 4 study drugs was not statistically significant due to the small number of events in this group. Further prospective studies of combination perioperative aspirin, beta-blockers, statins, and ACE inhibitors are warranted. Journal of Hospital Medicine 2010;5:218-225. @ 2010 Society of Hospital Medicine.

\section{KEYWORDS: mortality, perioperative medicine, vascular surgery, veterans.}

Additional Supporting Information may be found in the online version of this article.

Vascular surgery is the most morbid of the noncardiac surgeries, with a 30 -day mortality estimated to be $3 \%$ to $10 \%$ and 6-month mortality estimated to be $10 \%$ to $30 \% .^{1-4}$ Adverse outcomes are highly correlated with the presence of perioperative ischemia and infarction. Perioperative ischemia is associated with a 9-fold increase in the odds of unstable angina, nonfatal myocardial infarction, and cardiac death, while a perioperative myocardial infarction increases the odds of death 20 -fold up to 2 years after surgery. ${ }^{5-7}$ Prior research has centered on the single or combination use of perioperative beta-blockers and statins, which has been associated with decreased short-term and long-term mortality after vascular surgery, ${ }^{8-14}$ with the exceptions of the Metoprolol After Vascular Surgery (MAVS) ${ }^{15}$ and the Perioperative Beta-Blockade (POBBLE) studies, ${ }^{16}$ which were negative beta-blocker randomized controlled trials exclusively in vascular surgery patients, and the Perioperative Ischemic Evaluation (POISE) study, ${ }^{17}$ which was the largest perioperative beta-blocker trial to date in noncardiac surgery, with $41 \%$ of the patients undergoing vascular surgery.

There have been few studies assessing clinical outcomes in patients taking multiple concurrent cardioprotective 
medications. Clinicians are challenged to apply research results to their patients, who generally take multiple drugs. A retrospective cohort study of acute coronary syndrome patients did assess the use of evidence-based, combination therapies, including aspirin, ACE inhibitors, beta-blockers, and statins, compared to the use of none of these agents and found an association with decreased 6-month mortality. ${ }^{18}$ There are no prior noncardiac surgery studies assessing the concurrent use of multiple possibly cardioprotective drugs. There is 1 cohort study of coronary artery bypass graft surgery patients that assessed aspirin, ACE inhibitor, beta-blocker, and statin use and found associations with decreased mortality. ${ }^{19}$ As preoperative coronary revascularization has not been found to produce improved survival after vascular surgery, clarifying which perioperative medicines alone or in combination may improve outcomes becomes even more important. ${ }^{20}$ We sought to ascertain if the use of concurrent combination aspirin, ACE inhibitors, beta-blockers, and statins compared to nonuse was associated with a decrease in 6month mortality after vascular surgery.

\section{Patients and Methods}

\section{Setting and Subjects}

All patients presenting for vascular surgery at 5 regional Department of Veterans Affairs (VA) medical centers between January 1998 and March 2005 (3062 patients) were eligible for study entry. Patients with less than 6 months follow-up were excluded (42 patients). The study included the remaining 3020 patients (comprising 99\% of the original population). Our methods have been previously described. ${ }^{8}$ In brief, we conducted a retrospective cohort study using a regional VA administrative and relational database containing information on both the outpatient and inpatient environments. A record is generated for every contact a patient makes with the VA healthcare system, including prescription medications, laboratory values, demographic information, International Classification of Diseases, 9th Revision (ICD-9) codes, and vital status. In addition, we used the national VA death index, the VA Beneficiary Identification and Records Locator Subsystem database, which includes Social Security Administration data, to assess vital status. A patient was considered to have a drug exposure (aspirin, ACE inhibitor, beta-blocker, or statin) if the patient filled or renewed a prescription for the drug within 30 days before surgery. It was determined how many of these drugs were taken during this period, and in which combinations. The Institutional Review Board (IRB) at the Portland VA Medical Center approved the study with a waiver of informed consent.

\section{Data Elements}

For every patient we noted the type of vascular surgery (carotid, aortic, lower extremity bypass, or lower extremity amputation), age, sex, comorbid conditions (hypertension, cerebrovascular disease, cancer, diabetes, hyperlipidemia, chronic obstructive pulmonary disease [COPD], chronic kid- ney disease $[\mathrm{CKD}]$, coronary artery disease [CAD], or heart failure), nutritional status (serum albumin), and other medication use (also defined as filling a prescription within 30 days before surgery [insulin and clonidine]). Insulin use was documented to calculate the revised cardiac risk index (RCRI), ${ }^{21}$ and clonidine was documented to account for as a confounder. $^{22}$ The RCRI was assigned to each patient. One point was given for each of the following risk factors: use of insulin, CAD, heart failure, cerebrovascular disease, CKD, and high-risk surgery (intrathoracic, intraperitoneal, or suprainguinal vascular procedures). These variables were defined by ICD-9 codes. CKD was defined as either an ICD9 code for $\mathrm{CKD}$ or a serum creatinine $>2 \mathrm{mg} / \mathrm{dL}$. Patients were identified by the index vascular surgery using ICD-9 codes in the VA database, and data were extracted from both the inpatient and outpatient environments.

\section{Statistical Analysis}

Patients were included in the analysis if they either died within 6 months or were followed for at least 6 months. Data management and analyses were performed using SAS software, version 9.0. We conducted the univariate analysis of 6month mortality using chi-square analysis and provided unadjusted relative risk estimates for demographic and clinical variables. Demographic variables included age, sex, year, and site of surgery. Clinical variables included preoperative use of insulin and clonidine, preoperative medical conditions, serum albumin, creatinine, RCRI score, and type of surgery.

Bias due to confounding is a problem for studies that cannot randomize subjects into treatment groups. This bias can often be reduced by adjusting for the potentially confounding variables as covariates in regression models. However, when the number of potential confounders is large, as it was in our study, and the number of events, ie, deaths, is small, the resulting regression model can be unstable and the estimates unreliable. ${ }^{23,24}$ In such cases, it is necessary to control for confounding using another method. We chose to use propensity scoring and stratification analyses since these methods enable controlling for a large number of covariates using a single variable.

The study drugs were: aspirin, beta-blockers, statins, and ACE inhibitors. There are 16 combinations with 120 pairwise statistical comparisons possible for these 4 drug exposures. Instead of these multiple comparisons, we chose 4 classifications of combination drug exposure to examine: all 4 drugs compared to none, 3 drugs compared to none, 2 drugs compared to none, and 1 drug compared to none. Four different propensity scores were generated since we studied 4 different drug exposure classes. For each drug exposure class, propensity analyses were performed by using logistic regression to predict the likelihood of use of the drug of interest using all potential demographic and clinical confounding variables. Each subject received a score corresponding to the probability of their having a drug exposure based on the covariates. Scores were divided into quintiles, 
and these quintiles were used for stratification in CochranMantel-Haenszel analyses. Thus, we were able to test the association of patient survival to 6 months with the category of drug exposure comparisons within 30 days before surgery, while controlling for all aforementioned potential confounders. Results of the Breslow-Day test for homogeneity indicated that no statistically significant differences existed between the results of the propensity quintiles, so the overall summary statistic was reported. All quintiles achieved a balance in the covariates. However, for the 4 study drug exposure class, there were no deaths for the first $(\mathrm{n}=173)$ and second ( $\mathrm{n}=176$ ) quintiles (corresponding to lower-risk patients). We therefore excluded these patients from the final analysis.

Variables used in propensity scores included: age, sex, preoperative medical conditions, preoperative clonidine use, nutritional status (serum albumin), RCRI score, and year and location of surgery. To determine whether the propensity score adjustment removed imbalance among the comparisons of the combination drug classes to the no-drug-exposure patients, we evaluated associations between each classification of study drug exposure and predictor variables as compared to no-drug-exposure patients with both unadjusted chi-square and propensity-adjusted Cochran-MantelHaenszel analyses.

\section{Results}

\section{Patient Characteristics}

There were 3020 patients with a median age of 67 years, and interquartile range of 59 to 75 years. Ninety-nine percent were male, and all patients were assessed for death at 6 months after surgery (Table 1). Ten percent (304) had combination all-4-drug exposure, 22\% (652) had 3-drug exposure, 24\% (736) had 2-drug exposure, 26\% (783) had 1drug exposure, and $18 \%$ (545) had no study drug exposures. Eight percent (229) of surgeries were aortic, 28\% (861) were carotid, $28 \%$ (852) were lower extremity amputation, and $36 \%$ (1078) were lower extremity bypass. Twenty-two percent (665) of patients were low risk, with a RCRI of $0,60 \%$ (1822) were moderate risk with a RCRI of 1 to 2 , and $18 \%$ (553) were high risk with a RCRI of $\geq 3$. Overall the 6 -month mortality was $9.7 \%$ (294). The 6-month mortality for carotid endarterectomy was $5.0 \%$ (43/861), for lower extremity bypass $7.6 \%(82 / 1078)$, for aorta repair $9.2 \%(21 / 229)$, and for lower extremity amputation $17.4 \%$ (148/852).

The most common single-drug exposure was aspirin, $14 \%$ (416), followed by ACE inhibitors, 5\% (163) (Table 2). The more common 2-drug exposures included ACE inhibitors and aspirin, 7\% (203), aspirin and beta-blockers, 5\% (161), and aspirin and statins, 5\% (141). The common 3drug combinations included aspirin, beta-blockers, and statins, 8\% (229); ACE inhibitors, aspirin, and statins, 6\% (167); and ACE inhibitors, aspirin, and beta-blockers, 5\% (152). ACE inhibitor exposure was common in all combinations, eg, $20.8 \%$ of the 1-drug group had exposure to an ACE in- hibitor, $40.5 \%$ in the 2-drug group, $64.9 \%$ in the 3 -drug group, and all patients in the 4-drug group. Overall, 39.3\% of patients in the study had ACE inhibitor exposure. The gross unadjusted mortality for each drug exposure group was $10.6 \%$ for the no drug group, $11.2 \%$ for the 1 -drug group, $10.1 \%$ for the 2 -drug group, $8 \%$ for the 3 -drug group, and $7.2 \%$ for the 4 -drug group.

During the 6 complete years of the study (1998-2004) the frequency of combination exposure for all 4 study drugs increased from $3.5 \%$ to $13.4 \%$; 3-drug exposure also increased, $14.7 \%$ to $27.8 \%$; 2 -drug exposure remained relatively stable, $24.5 \%$ to $22 \%$; and single-drug exposure declined, $24.9 \%$ to $12.7 \%$ (Figure 1). Individual study drug exposures over the 6 years of the study generally also increased with respect to the other combinations: ACE inhibitor use increased, $34.5 \%$ to $42.5 \%$; beta-blocker, $27.8 \%$ to $53.4 \%$; statin, $22.6 \%$ to $52.2 \%$. The exception was aspirin, which was relatively stable, $54.5 \%$ in 1998 , and $57.2 \%$ in 2004 (Figure 2).

We also compared the use of the study drug exposures at 6 months after surgery to use within 30 days before surgery (Table 2). In the VA healthcare system aspirin is cheaper for some patients to purchase over-the-counter. Aspirin is likely underestimated in this dataset. The frequency of follow-up drug exposure at 6 months was overall similar to the drug exposure within 30 days before surgery. When aspirin was 1 of the combination exposures, the frequencies declined, and when aspirin was not 1 of the exposures, the frequencies generally increased. The frequency of no-drug exposures increased from $18.1 \%$ before surgery to $24.5 \% 6$ months after surgery, and the frequency of all 4 drug exposures decreased from $10.1 \%$ to $6.1 \%$, respectively.

\section{Univariate Analysis}

There were statistically significant differences in 6-month mortality for the combination drug exposure classes compared to no-drug exposure; $P$ value for linear trend $=0.018$ (Table 1).

\section{Propensity-adjusted Analysis}

Patients categorized in each combination drug exposure group were significantly different in their demographic and clinical characteristics compared to the no-drug exposure patients using unadjusted chi-square $P$ values (Appendix Table 1). However, after the propensity adjustments, only hyperlipidemia was statistically different for the combination 4-drug exposure patients compared to no-drug exposure patients (Appendix Table 1). All other demographic and clinical characteristics for the comparison of the drug exposure classes to no-drug exposure patients had statistically nonsignificant propensity-adjusted $P$ values. The range of propensity score distribution was fairly comparable for each combination drug exposure group. The Breslow-Day test for homogeneity was not significant among the quintiles for any of the drug exposure classes (Table 3; Appendix Table 


\section{TABLE 1. Patient Demographics and Unadjusted Relative Risk of 6-month Mortality}

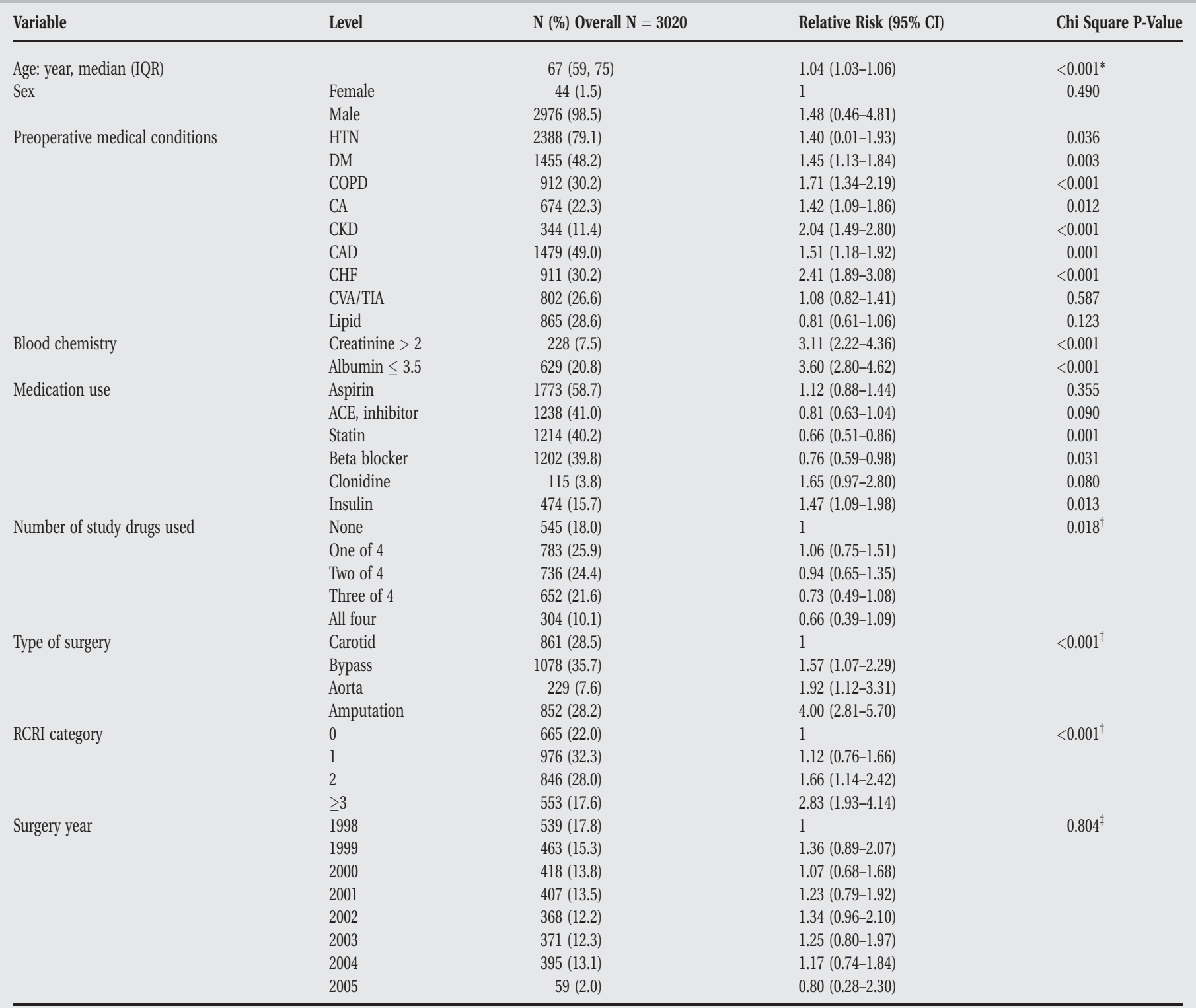

NOTE: Overall 6 month mortality: 9.7\% (294).

Abbreviations: Ace, angiotensin-converting enzyme; CA, cancer; CAD, coronary artery disease; CHF, congestive heart failure; CKD, chronic kidney disease; COPD, chronic obstructive pulmonary disease; CVA, cerebral vascular disease; DM, diabetes mellitus; HTN, hypertension; IQR, interquartile range; Lipid, hyperlipidemia; RCRI, Revised cardiac risk index; TIA, transient ischemic attack.

*Age was continuous.

${ }^{\dagger} \mathrm{P}$ for linear trend.

${ }^{\ddagger}$ Chi-square for overall group effect.

2), indicating that there was not a statistically significant difference in stratum-specific relative risks between the different quintiles. Therefore, the summary adjusted result was reported for each drug exposure group. Patients with all 4 drug exposures (with the first $[\mathrm{n}=173$ ] and second [ $\mathrm{n}=$ 166] quintiles excluded due to zero deaths) compared to nodrug exposure patients had a marginally significant association with decreased mortality, overall propensity-adjusted relative risk (aRR) 0.52 (95\% confidence interval [CI], 0.26-
1.01; $P=0.052$ ), number needed to treat (NNT) 19; patients with the combination 3-drug exposure had a significant association with decreased mortality, aRR 0.60 (95\% CI, 0.38-0.95; $P=0.030$ ), NNT 38 ; as well as patients with combination 2-drug exposure, aRR 0.68 (95\% CI, 0.46-0.99; $P=0.043$ ), NNT 170 (Table 3). Patients with 1 drug exposure did not have an association with decreased mortality compared to no-drug exposure patients, aRR 0.88 (95\% CI, $0.63-1.22 ; P=0.445)$. 


\begin{tabular}{|c|c|c|c|c|}
\hline \multirow[b]{2}{*}{ Drugs Used } & \multicolumn{2}{|c|}{ Presurgery } & \multicolumn{2}{|c|}{$\begin{array}{l}6 \text { Months } \\
\text { Postsurgery }\end{array}$} \\
\hline & Frequency & $\%$ & Frequency & $\%$ \\
\hline None & 545 & 18.1 & 669 & 24.5 \\
\hline \multicolumn{5}{|l|}{1 Drug } \\
\hline Aspirin & 416 & 53.1 & 169 & 28.3 \\
\hline ACE inhibitor & 163 & 20.8 & 135 & 22.6 \\
\hline Beta-blocker & 110 & 14.1 & 163 & 27.2 \\
\hline Statin & 94 & 12.0 & 131 & 21.9 \\
\hline All 1 drug & 783 & 100.0 & 598 & 100.0 \\
\hline \multicolumn{5}{|l|}{2 Drugs } \\
\hline Aspirin + ACE inhibitor & 203 & 27.6 & 102 & 14.4 \\
\hline Aspirin + Beta-blocker & 161 & 21.8 & 117 & 16.5 \\
\hline Aspirin + Statin & 141 & 19.2 & 86 & 12.1 \\
\hline ACE inhibitor + Beta-blocker & 56 & 7.6 & 103 & 14.5 \\
\hline ACE inhibitor + Statin & 89 & 12.1 & 126 & 17.7 \\
\hline Beta-blocker + Statin & 86 & 11.7 & 176 & 24.8 \\
\hline All 2 drugs & 36 & 100.0 & 710 & 100.0 \\
\hline \multicolumn{5}{|l|}{3 Drugs } \\
\hline Aspirin + ACE inhibitor + Beta-blocker & 152 & 23.3 & 96 & 16.5 \\
\hline Aspirin + ACE inhibitor + Statin & 167 & 25.6 & 103 & 17.7 \\
\hline Aspirin + Beta- blocker + Statin & 229 & 35.1 & 165 & 28.4 \\
\hline ACE inhibitor + Beta-blocker Statin & 104 & 16.0 & 218 & 37.4 \\
\hline All 3 drugs & 652 & 100.0 & 582 & 100.0 \\
\hline All 4 drugs & 304 & 10.1 & 167 & 6.1 \\
\hline Total & 3020 & 100.0 & $2726^{*}$ & 100.0 \\
\hline
\end{tabular}

\section{Discussion}

This retrospective cohort study has demonstrated that the combination use of 4 drugs (aspirin, beta-blockers, statins, and ACE inhibitors) compared to the use of none of these drugs had a trend toward decreased mortality, with a $49 \%$ decrease in propensity-adjusted 6-month mortality after vascular surgery and an NNT of 19. In addition, the combination use of 3 drug exposures was significantly associated with a $40 \%$ decrease in mortality, with propensity adjustment and NNT of 38; and the 2-drug combination exposure showed a significant association, with a propensity-adjusted $32 \%$ decreased mortality, and an NNT of 170 . Both the unadjusted and adjusted analyses showed a linear trend, suggesting a dose-response effect of more study-drug exposure association with less 6-month mortality and smaller NNT.

The lack of statistical significance for the 4-drug exposure group is likely due to few patients and events in this group, and the exclusion of the first 2 quintiles $(n=339)$ due to having zero deaths with which to compare. It is not unusual to exclude patients from analyses in propensity methods. The patients we excluded were low-risk who had survived to 6-months after surgery, so they would have also been excluded in a propensity-matched analysis. We did not per-

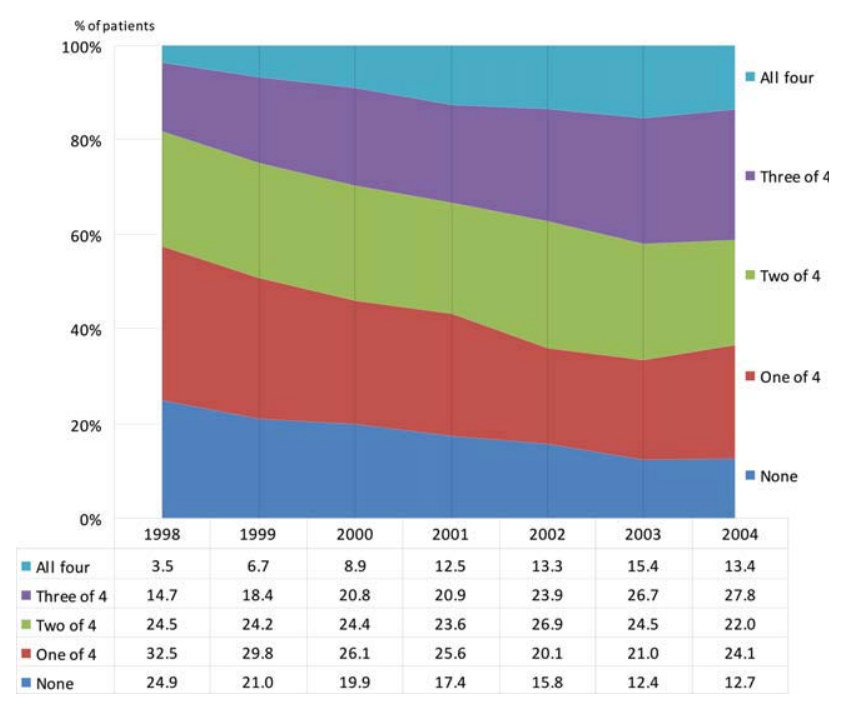

FIGURE 1. Frequency of combination study drug exposure classes over time.

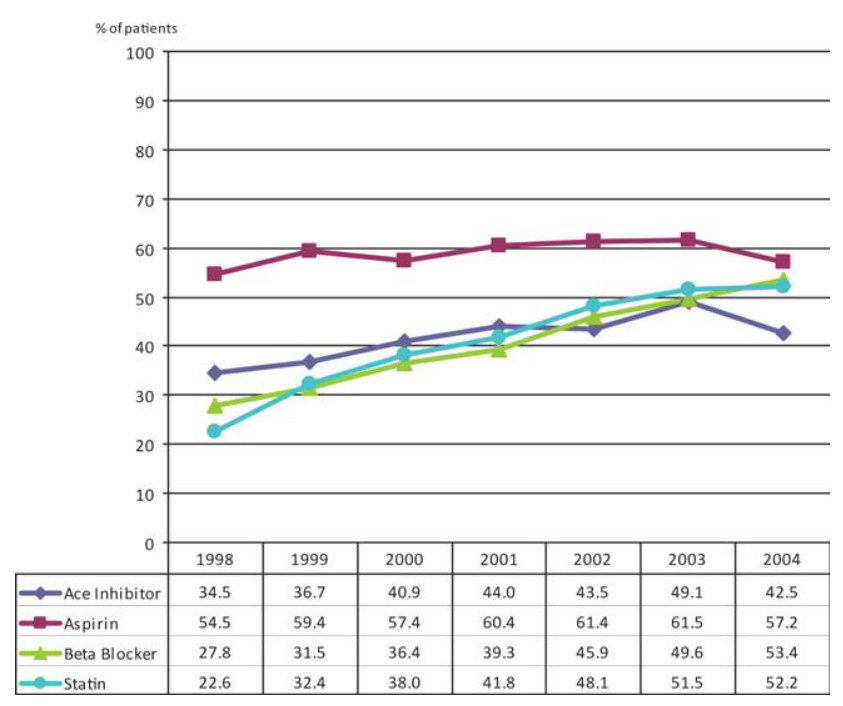

FIGURE 2. Frequency of individual study drugs over time.

form propensity matching, as we had adequate homogeneity between our quintile strata, and were not powered to perform matching.

This is the first evidence of which we are aware of an association with decreased mortality for the combination perioperative use of aspirin, beta-blockers, statins, and ACE inhibitors in vascular surgery patients. Aspirin has been associated with decreased mortality in patients undergoing coronary artery bypass graft surgery, ${ }^{25}$ but the effects of aspirin on noncardiac surgery outcomes is less clear. ${ }^{26}$

Beta-blockers and statins have been associated with decreased short-term and long-term mortality after vascular surgery in the past, ${ }^{8-14}$ but more recent beta-blocker studies have been negative, introducing controversy for the topic. ${ }^{15-17,27}$ Beta-blockers are currently recommended as: Class I (should be used), Evidence Level B (limited 
TABLE 3. Propensity-adjusted Associations of Drug Exposure Classes With 6-month Mortality

\begin{tabular}{|c|c|c|c|c|c|c|c|c|}
\hline \multirow[b]{2}{*}{ Variable } & \multirow[b]{2}{*}{$\mathrm{N}($ Overall $\mathrm{N}=3020)$} & \multicolumn{4}{|c|}{6 Mo. Mortality } & \multirow[b]{2}{*}{ P Value* } & \multirow{2}{*}{$\begin{array}{l}\text { Adjusted Relative } \\
\text { Risk (95\% CI) of Death* }\end{array}$} & \multirow[b]{2}{*}{$\mathrm{NNT}^{\dagger}$} \\
\hline & & $\%$ & $(\mathrm{n} / \mathrm{N})$ & $\%$ & $(\mathrm{n} / \mathrm{N})$ & & & \\
\hline 1 Drug vs. no drugs & 1328 & 10.64 & $(58 / 545)$ & 11.24 & $(88 / 783)$ & 0.445 & $0.88(0.63-1.22)$ & - \\
\hline 2 Drugs vs. no drugs & 1281 & 10.64 & $(58 / 545)$ & 10.05 & $(74 / 736)$ & 0.043 & $0.68(0.46-0.99)$ & 170 \\
\hline 3 Drugs vs. no drugs & 1197 & 10.64 & $(58 / 545)$ & 7.98 & $(52 / 652)$ & 0.030 & $0.60(0.38-0.95)$ & 38 \\
\hline
\end{tabular}

population risk strata evaluated) for vascular surgery patients already taking a beta-blocker or with positive ischemia on stress testing; Class IIa (reasonable to use), Evidence Level B for 1 or more clinical risk factors; or Class IIb (may be considered), Evidence Level B for no clinical risk factors, in the 2007 American College of Cardiology/American Heart Association (ACC/AHA) guidelines for perioperative evaluation. ${ }^{28}$ Perioperative beta-blocker trials that have titrated the dose to a goal heart rate have consistently been associated with improved outcomes after vascular surgery, ${ }^{10,12,29,30}$ and perioperative beta-blocker trials that have used fixed dosing after surgery have been negative, ${ }^{15-17,27}$ including the POISE trial, which was associated with increased strokes and mortality.

This is also the first evidence of which we are aware that ACE inhibitors in combination with other drugs may be associated with decreased mortality after vascular surgery. While our study design does not support a causal relationship between ACE inhibitor exposure and decreased mortality, the increasing exposure in each drug exposure group for ACE inhibitors and correlated decreasing mortality is of sufficient interest to warrant further study. The use of ACE inhibitors has been associated with decreased mortality in patients with atherosclerotic vascular disease and CAD. ${ }^{31}$ There has been a concern expressed in the literature about the perioperative use of ACE inhibitors due to the potential for intraoperative hypotension. ${ }^{32-36}$ Many centers advise patients to discontinue ACE inhibitor use the day before surgery. The number of patients studied remains small. More research is needed to clarify this issue. Use of angiotensin-receptor blockers was not assessed; their use was considered to be rare, because use was restricted to patients intolerant of ACE inhibitors during the study period.

The 2005 ACC/AHA guideline for patients with peripheral arterial disease recommends the use of aspirin and statins. ${ }^{37}$ ACE inhibitors are recommended for both asymptomatic and symptomatic peripheral artery disease patients. The 2006 ACC/AHA guidelines for secondary prevention for patients with coronary or other atherosclerotic vascular disease recommends the use of chronic beta-blockers. ${ }^{38}$ There appears to be some benefit in mortality from the combination aspirin, beta-blocker, statin, and ACE inhibitor drug regimen in patients with established atherosclerotic vascular disease.

We expect the frequency of aspirin exposure to be underestimated in this study population (due to over-the-counter undocumented use), so our findings may be somewhat underestimated as well. This may also explain why the frequency of aspirin remained constant over time while the other drug exposures increased over time.

Our study has several limitations. First, our design was a retrospective cohort. Propensity analysis attempts to correct for confounding by indication in nonrandomized studies as patients that are exposed to a study drug are different from patients that are not exposed to the same study drug. For example, without adjustment for the propensity scores, the drug exposure classes were significantly associated with demographic and clinical characteristics when compare to the no-drug-exposure patients. However, with the propensity score adjustment, these associations were no longer statistically significant, with the exception of hyperlipidemia in patients taking all 4 drugs, which supports a rigorous propensity adjustment. We also controlled for the use of clonidine and serum albumin, both strong predictors of death after noncardiac surgery. ${ }^{22,39}$ Second, we utilized administrative ICD-9 code data for abstraction, and utilized only documented and coded comorbidities in the VA database. Unmeasured confounders may exist. Further, we cannot identify which combinations of specific study drugs were most associated with a reduction in 6-month mortality, but we believe our data supports the case that all 4 of the study drugs be considered for each patient undergoing vascular surgery. It is important to also note that patient baseline risk, which can be difficult to clarify in retrospective cohort studies, will have a large impact on the results of the NNT. Lastly, this study needs to be repeated in a population that includes a greater number of female participants.

The combination exposure of 2 to 3 study drugs: aspirin, beta-blockers, statins, and ACE inhibitors was consistently associated with decreased 6-month mortality after vascular 
surgery, with a high prevalence of ACE inhibitor use, and the combination exposure of all 4 study drugs was marginally associated with decreased mortality. Consideration for the individual patient undergoing vascular surgery should include whether or not the patient may benefit from these 4 drugs. Further research with prospective and randomized studies is needed to clarify the optimum timing of these drugs and their combination efficacy in vascular surgery patients with attention to patient-specific risk.

\section{Acknowledgement}

The authors thank Martha S. Gerrity, MD, PhD, Portland VA Medical Center, Portland, Oregon, for comments on an earlier version of the manuscript.

\section{Address for correspondence and reprint requests:}

Thomas W. Barrett, MD, MCR, FHM, Portland VAMC (P3MED), 3710 SW US VA Hospital Road, Portland, OR 97207-1034; Telephone: 503-273-5015; Fax: 503-721-7807; E-mail:

barretth@ohsu.edu Received 9 September 2008; revision received 3 August 2009; accepted 26 August 2009.

\section{REFERENCES}

1. Feinglass J, Pearce WH, Martin GJ, et al. Postoperative and late survival outcomes after major amputation: findings from the Department of Veterans Affairs National Surgical Quality Improvement Program. Surgery. 2001;130(1):21-29.

2. Fleisher LA, Eagle KA, Shaffer T, Anderson GF. Perioperative- and longterm mortality rates after major vascular surgery: the relationship to preoperative testing in the Medicare population. Anesth Analg. 1999;89(4): 849-855.

3. Mays BW, Towne JB, Fitzpatrick CM, et al. Women have increased risk of perioperative myocardial infarction and higher long-term mortality rates after lower extremity arterial bypass grafting. J Vasc Surg. 1999;29(5): 807-812; discussion 12-13.

4. McFalls EO, Ward HB, Santilli S, Scheftel M, Chesler E, Doliszny KM. The influence of perioperative myocardial infarction on long-term prognosis following elective vascular surgery. Chest. 1998;113(3):681-686.

5. Mangano DT, Browner WS, Hollenberg M, London MJ, Tubau JF, Tateo IM. Association of perioperative myocardial ischemia with cardiac morbidity and mortality in men undergoing noncardiac surgery. The Study of Perioperative Ischemia Research Group. N Engl J Med. 1990;323(26): 1781-1788.

6. Mangano DT, Hollenberg M, Fegert G, et al. Perioperative myocardial ischemia in patients undergoing noncardiac surgery\&mdash;I: Incidence and severity during the 4 day perioperative period. The Study of Perioperative Ischemia (SPI) Research Group. J Am Coll Cardiol. 1991;17(4): 843-850.

7. Mangano DT, Wong MG, London MJ, Tubau JF, Rapp JA. Perioperative myocardial ischemia in patients undergoing noncardiac surgery\&mdash;II: Incidence and severity during the 1st week after surgery. The Study of Perioperative Ischemia (SPI) Research Group. J Am Coll Cardiol. 1991; 17(4):851-857.

8. Barrett TW, Mori M, De Boer D. Association of ambulatory use of statins and beta-blockers with long-term mortality after vascular surgery. J Hosp Med. 2007;2(4):241-252.

9. Durazzo AE, Machado FS, Ikeoka DT, et al. Reduction in cardiovascular events after vascular surgery with atorvastatin: a randomized trial. $J$ Vasc Surg. 2004;39(5):967-975; discussion 75-76.

10. Mangano DT, Layug EL, Wallace A, Tateo I. Effect of atenolol on mortality and cardiovascular morbidity after noncardiac surgery. Multicenter Study of Perioperative Ischemia Research Group. N Engl J Med. 1996;335(23): 1713-1720.
11. Poldermans D, Bax JJ, Kertai MD, et al. Statins are associated with a reduced incidence of perioperative mortality in patients undergoing major noncardiac vascular surgery. Circulation. 2003;107(14):1848-1851.

12. Poldermans D, Boersma E, Bax JJ, et al. The effect of bisoprolol on perioperative mortality and myocardial infarction in high-risk patients undergoing vascular surgery. Dutch Echocardiographic Cardiac Risk Evaluation Applying Stress Echocardiography Study Group. $N$ Engl J Med. 1999;341(24):1789-1794.

13. Wallace A, Layug B, Tateo I, et al. Prophylactic atenolol reduces postoperative myocardial ischemia. McSPI Research Group. Anesthesiology. 1998; 88(1):7-17.

14. Ward PR, Leeper NJ, Kirkpatrick JN, Lang RM, Sorrentino MJ, Williams KA. The effect of preoperative statin therapy on cardiovascular outcomes in patients undergoing infrainguinal vascular surgery. Int J Cardiol. 2005; 104(3):264-268.

15. Yang H, Raymer K, Butler R, Parlow J, Roberts R. The effects of perioperative beta-blockade: results of the Metoprolol after Vascular Surgery (MaVS) study, a randomized controlled trial. Am Heart J. 2006;152(5): 983-990.

16. Brady AR, Gibbs JS, Greenhalgh RM, Powell JT, Sydes MR. Perioperative beta-blockade (POBBLE) for patients undergoing infrarenal vascular surgery: results of a randomized double-blind controlled trial. J Vasc Surg. 2005;41(4):602-609.

17. Devereaux PJ, Yang H, Yusuf S, et al. Effects of extended-release metoprolol succinate in patients undergoing non-cardiac surgery (POISE trial): a randomised controlled trial. Lancet. 2008;371(9627):1839-1847.

18. Mukherjee D, Fang J, Chetcuti S, Moscucci M, Kline-Rogers E, Eagle KA. Impact of combination evidence-based medical therapy on mortality in patients with acute coronary syndromes. Circulation. 2004;109(6): 745-749.

19. Goyal A, Alexander JH, Hafley GE, et al. Outcomes associated with the use of secondary prevention medications after coronary artery bypass graft surgery. Ann Thorac Surg. 2007;83(3):993-1001.

20. McFalls EO, Ward HB, Moritz TE, et al. Coronary-artery revascularization before elective major vascular surgery. $N$ Engl J Med. 2004;351(27): 2795-2804

21. Lee TH, Marcantonio ER, Mangione CM, et al. Derivation and prospective validation of a simple index for prediction of cardiac risk of major noncardiac surgery. Circulation. 1999;100(10):1043-1049.

22. Wijeysundera DN, Naik JS, Beattie WS. Alpha-2 adrenergic agonists to prevent perioperative cardiovascular complications: a meta-analysis. Am J Med. 2003;114(9):742-752.

23. Cepeda MS, Boston R, Farrar JT, Strom BL. Comparison of logistic regression versus propensity score when the number of events is low and there are multiple confounders. Am J Epidemiol. 2003;158(3):280-287.

24. D'Agostino RB Jr. Propensity score methods for bias reduction in the comparison of a treatment to a non-randomized control group. Stat Med. 1998;17(19):2265-2281.

25. Mangano DT. Aspirin and mortality from coronary bypass surgery. $N$ Engl J Med. 2002;347(17):1309-1317.

26. Tangelder MJ, Lawson JA, Algra A, Eikelboom BC. Systematic review of randomized controlled trials of aspirin and oral anticoagulants in the prevention of graft occlusion and ischemic events after infrainguinal bypass surgery. J Vasc Surg. 1999;30(4):701-709.

27. Juul AB, Wetterslev J, Gluud C, et al. Effect of perioperative beta blockade in patients with diabetes undergoing major non-cardiac surgery: randomised placebo controlled, blinded multicentre trial. Br Med J (Clin Res Ed). 2006;332(7556):1482.

28. Fleisher LA, Beckman JA, Brown KA, et al. ACC/AHA 2007 Guidelines on perioperative cardiovascular evaluation and care for noncardiac surgery: executive summary: a report of the American College of Cardiology/ American Heart Association Task Force on Practice Guidelines (Writing Committee to Revise the 2002 Guidelines on Perioperative Cardiovascular Evaluation for Noncardiac Surgery): developed in collaboration with the American Society of Echocardiography, American Society of Nuclear Cardiology, Heart Rhythm Society, Society of Cardiovascular Anesthesiologists, Society for Cardiovascular Angiography and Interventions, Society 
for Vascular Medicine and Biology, and Society for Vascular Surgery. Circulation. 2007;116(17):1971-1996.

29. Feringa HH, Bax JJ, Boersma E, et al. High-dose beta-blockers and tight heart rate control reduce myocardial ischemia and troponin $\mathrm{T}$ release in vascular surgery patients. Circulation. 2006;114(1 suppl):I344-I349.

30. Poldermans D, Bax JJ, Schouten O, et al. Should major vascular surgery be delayed because of preoperative cardiac testing in intermediate-risk patients receiving beta-blocker therapy with tight heart rate control? $J$ Am Coll Cardiol. 2006;48(5):964-969.

31. Yusuf S, Sleight P, Pogue J, Bosch J, Davies R, Dagenais G. Effects of an angiotensin-converting-enzyme inhibitor, ramipril, on cardiovascular events in high-risk patients. The Heart Outcomes Prevention Evaluation Study Investigators. N Engl J Med. 2000;342(3):145-153.

32. Brabant SM, Bertrand M, Eyraud D, Darmon PL, Coriat P. The hemodynamic effects of anesthetic induction in vascular surgical patients chronically treated with angiotensin II receptor antagonists. Anesth Analg. 1999; 89(6):1388-1392.

33. Colson P, Saussine M, Seguin JR, Cuchet D, Chaptal PA, Roquefeuil B. Hemodynamic effects of anesthesia in patients chronically treated with angiotensin-converting enzyme inhibitors. Anesth Analg. 1992;74(6): 805-808.

34. Comfere T, Sprung J, Kumar MM, et al. Angiotensin system inhibitors in a general surgical population. Anesth Analg. 2005;100(3):636-644.
35. Coriat P, Richer C, Douraki T, et al. Influence of chronic angiotensin-converting enzyme inhibition on anesthetic induction. Anesthesiology. 1994; 81(2):299-307.

36. Schirmer U, Schurmann W. Preoperative administration of angiotensinconverting enzyme inhibitors. Anaesthesist. 2007;56(6):557-561.

37. Hirsch AT, Haskal ZJ, Hertzer NR, et al. ACC/AHA 2005 Practice guidelines for the management of patients with peripheral arterial disease (lower extremity, renal, mesenteric, and abdominal aortic): executive summary\&mdash;a collaborative report from the American Association for Vascular Surgery/Society for Vascular Surgery, Society for Cardiovascular Angiography and Interventions, Society for Vascular Medicine and Biology, Society of Interventional Radiology, and the ACC/AHA Task Force on Practice Guidelines (Writing committee to develop guidelines for the management of patients with peripheral arterial disease). Circulation. 2006;113(11):1474-1547.

38. Smith SC Jr, Allen J, Blair SN, et al. AHA/ACC guidelines for secondary prevention for patients with coronary and other atherosclerotic vascular disease: 2006 update: endorsed by the National Heart, Lung, and Blood Institute. Circulation. 2006;113(19):2363-2372.

39. Gibbs J, Cull W, Henderson W, Daley J, Hur K, Khuri SF. Preoperative serum albumin level as a predictor of operative mortality and morbidity: results from the National VA Surgical Risk Study. Arch Surg. 1999;134(1): 36-42. 